\title{
A Floating-Gate Programmable Array of Silicon Neurons for Central Pattern Generating Networks
}

\author{
Francesco Tenore $^{1}$, R. Jacob Vogelstein ${ }^{2}$, Ralph Etienne-Cummings ${ }^{1}$, Gert Cauwenberghs ${ }^{3}$ and Paul Hasler ${ }^{4}$ \\ ${ }^{1}$ Department of Electrical and Computer Engineering, Johns Hopkins University, Baltimore, MD \\ ${ }^{2}$ Department of Biomedical Engineering, Johns Hopkins University, Baltimore, MD \\ ${ }^{3}$ Division of Biological Sciences, University of California San Diego, La Jolla, CA \\ ${ }^{4}$ Department of Electrical and Computer Engineering, Georgia Institute of Technology, Atlanta, GA \\ \{ftenore, jvogelst,retienne\}@jhu.edu, gert@ucsd.edu, paul.hasler@ee.gatech.edu
}

\begin{abstract}
A new central pattern generator chip with 24 silicon neurons and reprogrammable connectivity is presented. The $3 \mathrm{~mm} \times 3 \mathrm{~mm}$ chip fabricated in a $3 \mathrm{M} 2 \mathrm{P} 0.5 \mu \mathrm{m}$ process contains 1032 synapses, each with multiple floating gates for storing parameters governing synaptic strength and polarity. Every neuron includes a dendritic compartment with 12 externallyaddressable synaptic inputs and 24 recurrent synaptic inputs, enabling construction of a fully-interconnected network with sensory feedback from off-chip elements. In addition to describing the chip architecture and neuron circuits, preliminary results from single oscillating neurons and pairs of phase-locked neurons are shown. This work represents the realization of a design presented at ISCAS'05, and an improvement over our 2nd generation CPG chip presented at ISCAS'04.
\end{abstract}

\section{INTRODUCTION}

Central pattern generators (CPGs) are small, semiautonomous networks of neurons that produce rhythmic patterned outputs to control motor functions. CPGs have been found in all organisms studied, from invertebrates [1] to vertebrates [2] and, recently, humans [3]; they are responsible for behaviors such as flying, swimming, chewing, walking, breathing, and other regular activities [4], [5]. Although typically not necessary for basic functionality [6], sensory feedback and descending inputs from the brain are normally used to modulate and control the CPG output [7].

The goal of this work is to develop a silicon analog of the biological central pattern generator to control locomotion in robots and, potentially, to serve as an in vivo replacement for a real CPG after spinal cord injury. A number of silicon CPGs can be found in the literature [8]-[11] with varying degrees of biological realism. Chips with the most sophisticated neural models [8] are based on the Hodgkin-Huxley formalism and generate realistic spike outputs. However, these neurons occupy a large silicon area and are not well-suited to largescale integrated networks. The use of simple integrate-and-fire models allows for implementation of many neurons on the same silicon die [9], but forgoes the realistic dynamics that may be useful in generating complex output patterns.

Our previous CPG chip [9] allowed for 10 silicon neurons to be fully interconnected via digitally-controlled synapses. This architecture was used to create oscillatory networks with sufficient complexity to control a bipedal robot [12]. However, the digital-to-analog converters employed to store the synaptic

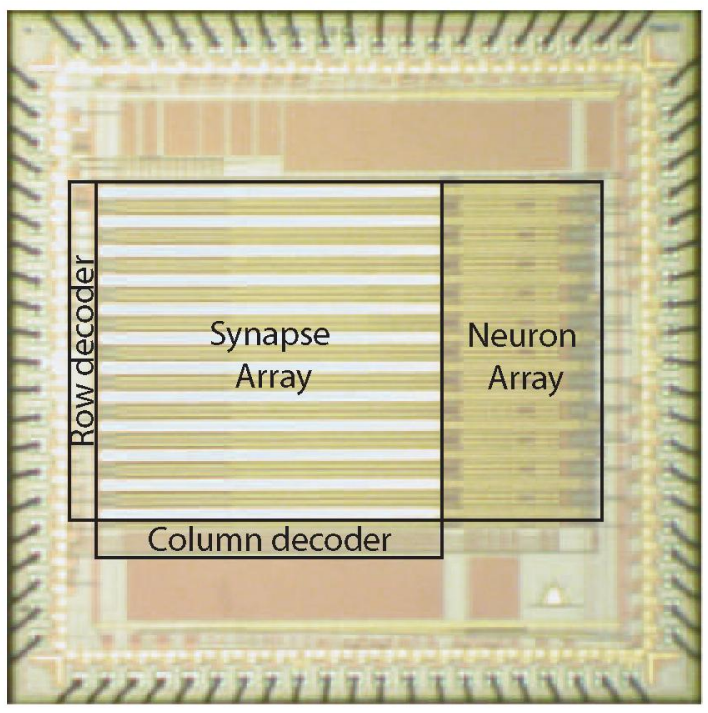

Fig. 1. Micrograph of the $3 \mathrm{~mm} \times 3 \mathrm{~mm}$ FG-CPG chip fabricated in a $3 \mathrm{M} 2 \mathrm{P}$ $0.5 \mu \mathrm{m}$ process.

strengths occupied a large on-chip area and required programming each time the chip was powered on. More recently, we presented a design for compact synapses using non-volatile analog storage on floating-gate (FG) transistors [13]. We have now fabricated and begun to test a chip (Fig. 1) that integrates 1032 of these FG synapses in an array of 24 silicon neurons.

\section{CHIP ARCHITECTURE}

The floating-gate central pattern generator (FG-CPG) chip architecture is similar to that described in [9], [13], and is illustrated in Figure 2. Twenty-four identical silicon neurons are arranged in rows, with 12 external inputs and 24 recurrent inputs running in columns across the chip. Each of the 36 inputs makes synaptic connections to all 24 neurons via the floating-gate synapse circuits described in Section II-A (Fig. 3). In addition to the external and recurrent inputs, neurons also receive a bias current from a simple current mirror (not shown).

\section{A. Neuron Circuit}

A block diagram of the neuron subcircuit is shown in Figure 4. As mentioned in [13], these cells have three differ- 


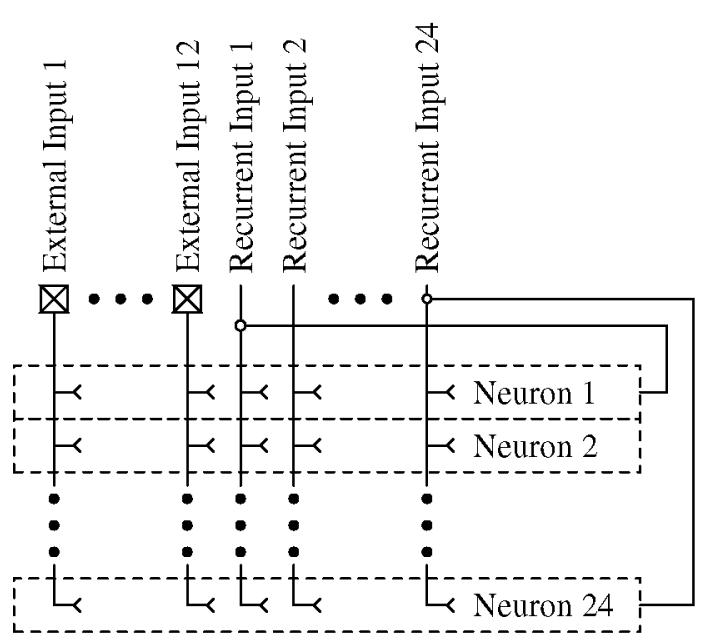

Fig. 2. The FG-CPG chip floorplan. Twenty-four silicon neurons are arranged in rows, and thirty-six inputs are arranged in columns. A floating-gate synapse is located at the intersection between each input and neuron. The first 12 inputs are "external" in the sense that they are gated by off-chip signals; the remaining 24 inputs are gated by the outputs of the 24 on-chip neurons and are therefore called "recurrent". A fully-interconnected network can be created by activating all of the recurrent synapses.

ent compartments-dendritic, somatic, and axonal-that are both functionally and spatially distinct. In Figure 1, it can be seen that the dendritic compartments (labeled "synapse array") occupy most of the silicon area, while the somatic and axonal compartments (labeled "neuron array") are relatively smaller. This is partially due to the large number of synapses implemented, but mostly because the membrane capacitance is distributed throughout the dendritic compartment to achieve a compact layout. The contents of the neuronal compartments are described briefly below:

- The dendritic compartment contains 12 external and 24 recurrent synapses, where each synapse is implemented with the floating-gate circuit described in Section II-B. For testing purposes, a bias current can also serve as an input to the neuron.

- The somatic compartment contains a large capacitor (modeling the membrane capacitance of a biological neuron), a hysteretic comparator (modeling the axon hillock), and some specialized "synapses" that discharge the cell and implement a refractory period.

- The axonal compartment contains specialized "synapses" that produce variable-duration spike outputs and allow for spike-frequency adaptation. The spike outputs are buffered and sent off-chip to control external motor systems, and are also used to gate the 24 recurrent synapses between each cell, all of its neighbors, and itself.

Although all three compartments of the neurons contain the same synapse circuit (Sec. II-B), only the 36 dendritic inputs are synapses in the traditional sense. Programmable current sources used to reset the neuron and implement a refractory period, spike-width modulation, and spike-frequency adaptation are implemented with a floating-gate synapse circuit only

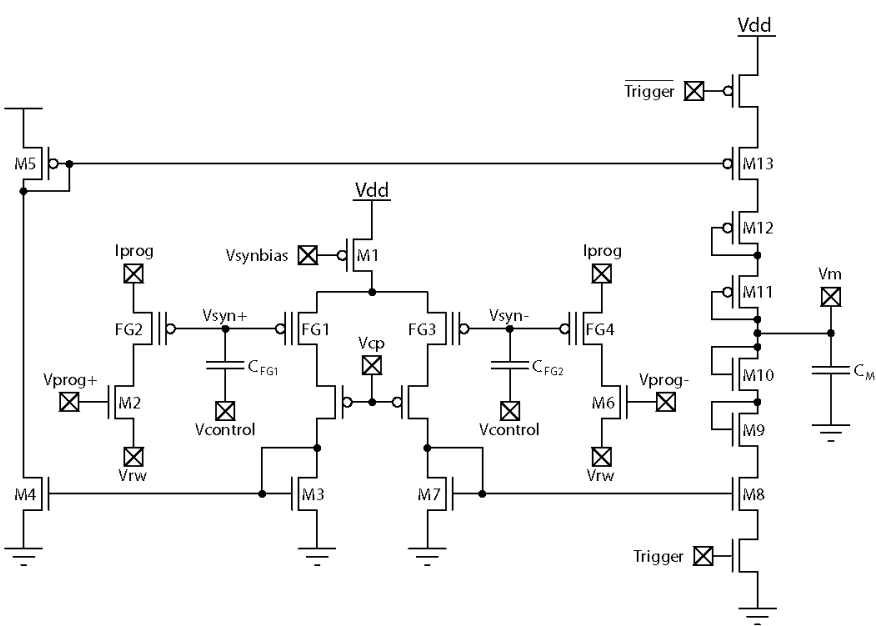

Fig. 3. Floating-gate synapse circuit schematic. Each synapse contains nonvolatile analog memory elements for storing a differential voltage on the gates of FG1-FG4, which determines the strength and polarity of the synapse.

for convenience.

\section{B. Synapse Circuit}

The 1032 on-chip floating-gate synapses (including external, recurrent, and specialized synapses) are all implemented with a simple nine-transistor operational transconductance amplifier (OTA), similar to that presented in [13] (Fig. 3). Briefly, hotelectron injection (HEI) [14] and Fowler-Nordheim tunneling [15] are used to add and remove charge from $\mathrm{C}_{\mathrm{FG} 1}$ and $\mathrm{C}_{\mathrm{FG}}$, creating a differential voltage for the OTA and thereby setting the current through M13 and M8. When the synapse is activated by placing a digital high voltage on Trigger and a low voltage on $\overline{\text { Trigger }}$, this differential current flows through $\mathrm{C}_{\mathrm{M}}$ and affects the membrane potential $V_{m}$. If $V_{s y n+}$ is less than $V_{\text {syn- }}$, the synapse will be excitatory and add charge to $\mathrm{C}_{\mathrm{M}}$; inhibitory synapses are created by setting $V_{s y n+}$ greater than $V_{s y n-}$. Because Fowler-Nordheim tunneling requires high voltages, HEI is used for most programming tasks - changes in synaptic strength and polarity are both achieved by varying the differential OTA voltage-and tunneling is reserved for "resetting" the synapses when the absolute voltage on a floating gate reaches zero. One notable improvement of this synapse circuit over the design presented in [13] is the addition of the $V_{\text {control }}$ node, which allows for increased programming range and better control of the voltage on the floating gates.

\section{RESULTS}

Figure 5 shows preliminary data recorded from a single onchip neuron. The neuron is oscillating due to a constant influx of bias current in its dendritic compartment. By adjusting the bias input, it is possible to achieve a wide range of spike frequencies, from approximately $1 \mathrm{~Hz}$ to $150 \mathrm{kHz}$. The maximum and minimum values of the membrane potential are set by the somatic compartment's hysteretic comparator; through appropriate tuning of the comparator's midpoint and tail current, it is possible to make the charging and discharging nonlinear due to the diode-connected transistors at the 


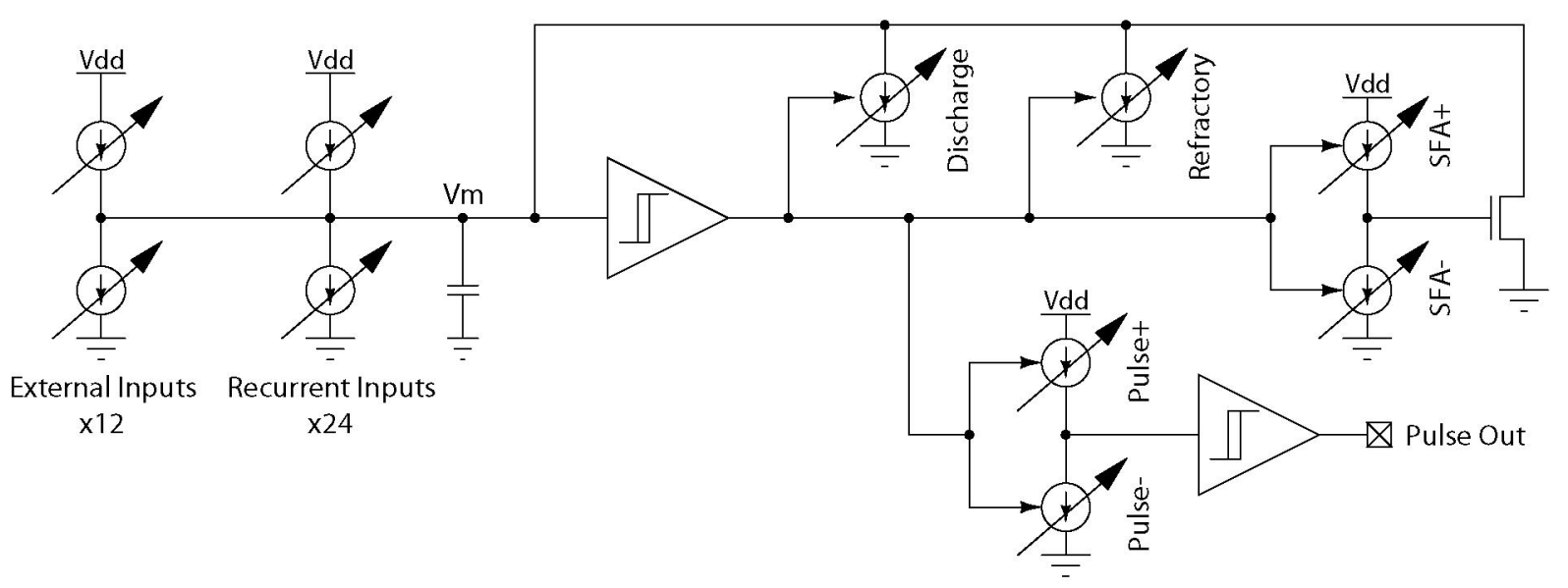

Fig, 4. Block diagram of our silicon neuron. Including the specialized "synapses" for discharging the cell and implementing a refractory period, pulse width modulation, and spike-frequency adaptation, each neuron contains 43 floating-gate synapses (drawn as an individual or pair of adjustable current sources).

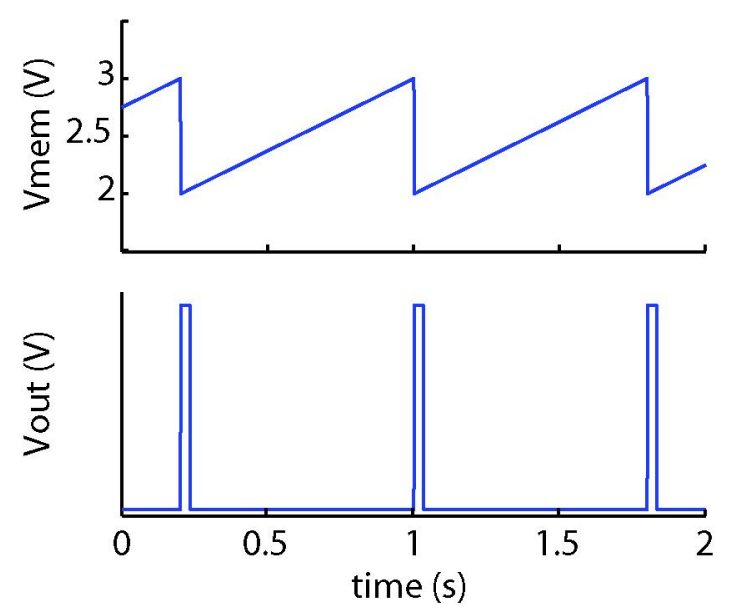

Fig. 5. A single neuron can be made to oscillate by injecting a small bias current onto its membrane capacitor. (Top) Oscilloscope output displaying neuron's membrane potential. (Bottom) Oscilloscope output displaying neuron's pulse outputs. The pulse width is relatively short here, but can be adjusted over a wide range of values by programming the specialized pulse width "synapse" (Sec. II-A).

synapses' output node (M9-M12 in Figure 3). The benefits of this nonlinearity have been explored in our previous work [9], [12], [13].

In order to generate useful signals for locomotion, a central pattern generator must be able to generate phase-locked outputs. Figure 6 illustrates two examples of phase-locking using neurons from the FG-CPG chip. In both cases, each neuron received excitatory input from an external synapse and inhibited the other neuron through a recurrent synapse. Phase delays were created by varying the synaptic strength of the external input. Figure 6 also shows an example of how the specialized pulse width "synapse" can be used to increase or decrease the duration of the neuron's output. The ability to create variable-duration outputs with arbitrary phase relationships is important when the CPG is used to control locomotion with multiple flexor/extensor pairs. For

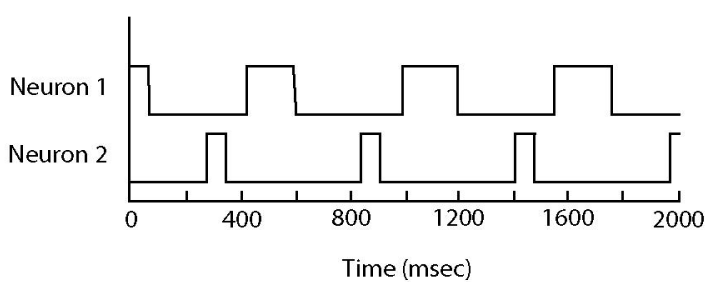

(a)

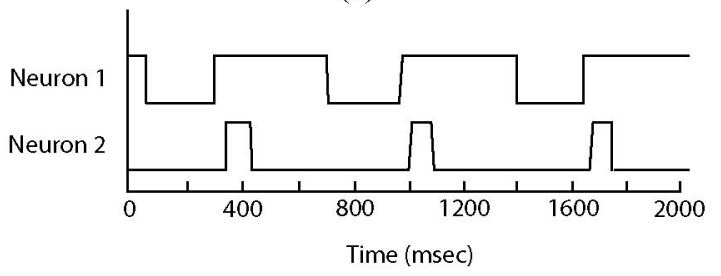

(b)

Fig. 6. Oscilloscope output displaying phase-locked neurons with different phase offsets and pulse widths.

example, the onsets of hip and knee actuators are typically $90^{\circ}$ degrees out-of-phase, and the duration of hip activation is approximately twice as long as knee activation [16].

\section{CONCLUSION}

Our preliminary results demonstrate the functionality of our 3rd generation central pattern generator chip. By replacing the bulky digital-to-analog converters in our previous design [9] with compact floating gate circuits, we were able to increase the number of neurons and synapses by $85 \%$ and $261 \%$, respectively, for an equivalent silicon area. Additionally, the chip is more versatile than the previous design: as the the tail currents on the synapse OTAs are varied from subthreshold currents to large suprathreshold currents, the neurons generate oscillations over six orders of magnitude in the frequency domain.

We are currently working to create complex networks sufficient to produce locomotion in a robotic biped. Previously, we presented a network of twelve neurons that can control 


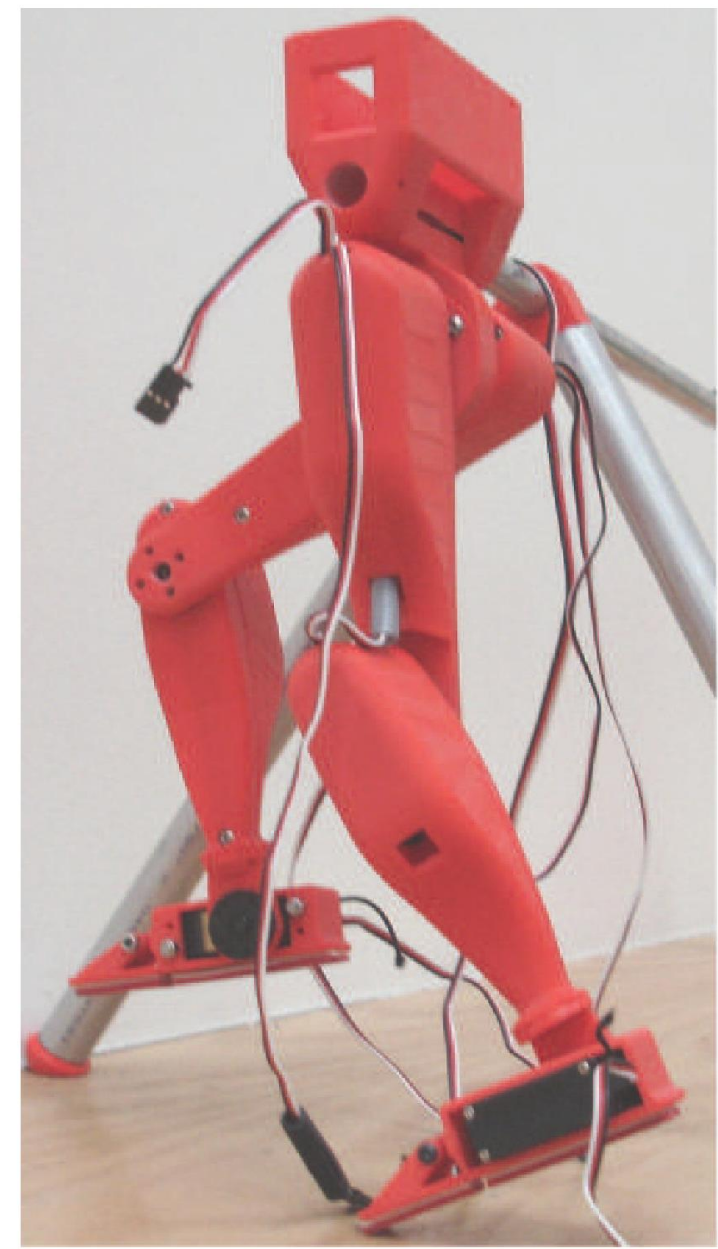

Fig. 7. The RedBot robot (courtesy Iguana Robotics Inc., Urbana, IL, USA),

bilateral hip and knee extensors and flexors [16], and can be extended to control robotic feet, as well (unpublished). Application of appropriate sensory feedback should allow for stable output patterns in the presence of perturbations such as those occurring during overground walking [17]. We therefore intend to implement this network on a single FG-CPG chip and use it to control the RedBot robot (Fig. 7).

\section{ACKNOWLEDGMENT}

The authors would like to thank the NSF-sponsored Telluride Neuromorphic Engineering Workshop for the precious insights it always offers and its invaluable learning experience. We would also like to thank Tony Lewis for his assistance with RedBot and for many helpful discussions on CPG-based control. This work was partially supported by NSF grant EEC9731478, ONR grant N00014-00-1-0562 and an NSF GRFP grant to RJV.

\section{REFERENCES}

[1] U. Bassler and A. Buschges, "Pattern generation for stick insect walking movements - multisensory control of a locomotor program," Brain Research Reviews, vol. 27, no. 1, pp. 65-88, 1998.

[2] S. Grillner, O. Ekeberg, A. E. Manira, A. Lansner, D. Parker, J. Tegner, and P. Wallen, "Intrinsic function of a neuronal network-a vertebrate central pattern generator," Brain Research Reviews, vol. 26, no. 2-3, pp. 184-197, 1998.

[3] M. R. Dimitrijevic, Y. Gerasimenko, and M. M. Pinter, "Evidence for a spinal central pattern generator in humans," Annals of the New York Academy of Sciences, vol. 860, pp. 360-376, 1998.

[4] E. Marder and D. Bucher, "Central pattern generators and the control of rhythmic movements"," Current Biology, vol. 11, no. 23, pp. R986R996, 2001.

[5] A. H. Cohen, S. Rossignol, and S. Grillner, Eds., Neural Control of Rhythmic Movements in Vertebrates. New York: Wiley, 1988.

[6] A. H. Cohen and P. Wallén, "The neuronal correlate of locomotion in fish. 'Fictive swimming' induced in an in vitro preparation of the lamprey spinal cord," Experimental Brain Research, vol, 41, pp. 11-18, 1980.

[7] A. Buschges, "Sensory control and organization of neural networks mediating coordination of multisegmental organs for locomotion," Journal of Neurophysiology, vol. 93, no, 3, pp. 1127-1135, 2005.

[8] M. F. Simoni, G. S. Cymbalyuk, M. E. Sorensen, R. L. Calabrese, and S. P. DeWeerth, "A multiconductance silicon neuron with biologically matched dynamics," IEEE Transactions on Biomedical Engineering, vol. 51, no. 2, pp. 342-354, 2004.

[9] F. Tenore, R. Etienne-Cummings, and M. A. Lewis, "A programmable array of silicon neurons for the control of legged locomotion," in IEEE International Symposium on Circuits and Systems, vol. 5, 2004, pp. V349-V352.

[10] K. Nakada, T. Asai, T. Hirose, and Y. Amemiya, "Analog CMOS implementation of a neuromorphic oscillator with current-mode lowpass filters," in IEEE International Symposium on Circuits and Systems, 2005, pp. 1923-1926.

[11] P. Arena, L. Fortuna, M. Frasca, L. Patane, and G. Vagliasindi, "CPGMTA implementation for locomotion control," in IEEE International Symposium on Circuits and Systems, 2005, pp. 4102-4105.

[12] F. Tenore, R. Etienne-Cummings, and M. A. Lewis, "Entrainment of silicon central pattern generators for legged locomotory control," in Advances in Neural Information Processing Systems 16, S. Thrun, L. Saul, and B. Schölkopf, Eds. Cambridge, MA: MIT Press, 2004.

[13] F. Tenore, R. J. Vogelstein, R. Etienne-Cummings, G, Cauwenberghs, M. A. Lewis, and P. Hasler, "A spiking silicon central pattern generator with floating gate synapses," in IEEE International Symposium on Circuits and Systems, 2005, pp, 4106-4109.

[14] C. Diorio, "A p-channel MOS synapse transistor with self-convergent memory writes," IEEE Transactions on Electron Devices, vol. 47, no. 2, pp. $464472,2000$.

[15] M. Lenzlinger and E. H. Snow, "Fowler-Nordheim tunneling into thermally grown SiO2," Journal of Applied Physics, vol, 40, no. 1, pp. 278-283, 1969.

[16] M. A. Lewis, F. Tenore, and R. Etienne-Cummings, "CPG design using inhibitory networks," in IEEE International Conference on Robotics and Automation, 2005.

[17] M. A. Lewis, R. Etienne-Cummings, M. H. Hartmann, A. H. Cohen, and Z. R. Xu, "An in silico central pattern generator: silicon oscillator, coupling, entrainment, physical computation \& biped mechanism control," Biological Cybernetics, vol. 88, no. 2, pp. 137-151, 2003. 